\title{
Diversité de la flore ligneuse à l'intérieur du Parc National de Waza (Cameroun)
}

\author{
Evaliste Rémi JIAGHO ${ }^{*}$, Louis-Paul-Roger KABELONG BANOHO ${ }^{2}$ et \\ Rodrigue Aimé FEUMBA ${ }^{1}$ \\ ${ }^{1}$ Département de Géographie, École Normale Supérieure, Université de Yaoundé 1, Cameroun. \\ ${ }^{2}$ Département de Biologie et de Physiologie Végétales, Université de Yaoundé 1, Cameroun. \\ *Auteur correspondant ; E-mail : remijiagho@yahoo.fr
}

Received: 10-03-2021

Accepted: $15-05-2021$

Published: 30-06-2021

\section{RESUME}

Le Parc National de Waza est une aire protégée de renommée internationale. Il est l'un des plus emblématiques que compte le Cameroun, mais fait face à des pressions anthropiques et d'origine naturelle qui menacent l'intégrité de ses ressources. La présente étude avait pour objectif d'améliorer la connaissance de la flore et de la végétation ligneuse afin de permettre de mieux bâtir les stratégies de gestion de ce parc. Pour y parvenir, un inventaire de la flore ligneuse a été réalisé en 2016, avec la méthode des transects couplée à celle des quadrats de $25 \mathrm{~m}$ x $50 \mathrm{~m}$. Le comptage et la mesure des paramètres dendrométriques, ainsi que les observations effectuées à l'intérieur de ces quadrats ont permis d'étudier les différents paramètres qualitatifs et quantitatifs de la ressource ligneuse, dont la richesse spécifique et les indices de diversité. Il en ressort que le parc abrite 38 espèces appartenant à 33 genres répartis dans 19 familles. L'indice de Shannon Weaver est de 2,95 et l'équitabilité de Piélou est de 0,56. Ces indices montrent que l'écosystème reste encore relativement riche, même si les menaces de la dégradation de ses ressources sont perceptibles. Il importe de mettre sur pied les stratégies efficaces de gestion de cette aire protégée et de sa périphérie afin de limiter les menaces auxquelles elle fait face.

(C) 2021 International Formulae Group. All rights reserved.

Mots clés : Inventaire floristique, écosystèmes, équitabilité, diversité, Parc National de Waza.

\section{Diversity of the woody plants species within the Waza National Park (Cameroon)}

\begin{abstract}
Waza National Park is an internationally renowned protected area. It is one of the most emblematic in Cameroon, but faces anthropogenic and natural pressures that threaten the integrity of its resources. The purpose of the study was to improve knowledge of the flora and woody vegetation in order to better build management strategies for this park. To achieve this, an inventory of the woody flora was carried out, using the transect method coupled with that of $25 \mathrm{~m}$ x $50 \mathrm{~m}$ quadrats. The counting and measurement of dendrometric parameters, as well as the observations made inside these quadrats, made it possible to study the various qualitative and quantitative
\end{abstract}


parameters of the woody resource, including specific richness and diversity indices. It appears that the park is home to 38 species belonging to 33 genera distributed in 19 families. The Shannon Weaver index is 2.95 and the Piélou fairness is 0.56 . These indices show that the ecosystem is still relatively rich, even if the threats of the degradation of its resources are perceptible. It is important to set up effective management strategies for this protected area and its periphery in order to limit the threats it faces.

(C) 2021 International Formulae Group. All rights reserved.

Keywords: Floral inventory; ecosystems, fairness, diversity, Waza National Park.

\section{INTRODUCTION}

Le Parc National de Waza (PNW), l'une des plus emblématiques aire protégée que compte le Cameroun (Wafo, 2008). Créé en 1934 sous forme de réserve et érigé en Parc National en 1968, il a, depuis sa création, connu des défis dus à sa gestion et à la conservation des ressources aussi bien faunique que floristique qu'il abrite. Pour les populations riveraines et de nombreux acteurs, la création du barrage de retenu de Maga en 1978 est la principale cause de la dégradation de cet écosystème, car, elle a entrainé l'assèchement de la plaine d'inondation de Waza Logone qui abrite ce parc. Suite à ce constat alarmant, l'Union Internationale pour la Conservation de la Nature (UICN) avait entrepris vers la fin des années 80 un programme de ré-inondation de la plaine grâce à la création des canaux. Après le projet de réinondation de la plaine, les résultats obtenus ont été mitigés du fait que, la dégradation du parc n'avait pas pour seule cause la création du barrage de Maga. De fait, penser que la dégradation de cet écosystème, d'une richesse particulière aussi bien faunique, floristique que piscicole, avait pour seule cause la création du barrage de Maga était simpliste. En réalité, la dégradation des écosystèmes soudanosahéliens connaissaient déjà une destruction de leurs ressources due aux facteurs naturels et aux activités anthropiques.

Depuis les années 1950, on a assisté à une dégradation des paysages soudanosahéliens d'Afrique occidentale et centrale. La cause principale se trouve dans la croissance démographique qui entraîne d'une part l'extension des surfaces cultivées, la disparition des jachères, la surcharge en bétail et l'épuisement des sols, et, d'autre part, l'émigration saisonnière ou de longue durée des populations. Ce constat a été fait par des travaux aussi bien au Cameroun qu'ailleurs (Mvondo et al., 2003 ; Jiagho, 2009 ; Kabelong Banoho, 2013; Djoufack-Manetsa, 2011; Jiagho et al., 2016 ; Jiagho, 2018 ; Mbaiyetom et al., 2021).

Face à ces constats de dégradation des ressources du PNW, il importe de mettre en place des stratégies idoines de conservation et de restauration. Cependant, la gestion durable d'une aire de conservation implique un certain nombre de préalables parmi lesquels la connaissance de la flore et de la végétation ligneuse. Malheureusement très peu d'aires protégées du Cameroun disposent de données fiables et actualisées sur la flore et la végétation (Jiagho et al., 2016). Pourtant, la connaissance de la biodiversité surtout végétale constitue une base pour des aménagements nécessaires à la restauration de l'environnement. Plusieurs auteurs (Kent et Coker, 2003 ; Cisse et al., 2020 ; Gnoumou et al., 2020) soutiennent d'ailleurs que les inventaires floristiques et les études des groupements végétaux sont la base incontournable pour l'établissement d'un plan de gestion et de conservation des écosystèmes.

Le Parc National de Waza est relativement peu connu sur le plan botanique. La taxonomie des espèces végétales réalisée jusqu'alors par Scholte et al. (2000) n'est que partielle et date de plus de quinze années. La récente étude botanique est celle de Jiagho et al. (2016), mais qui s'est focalisée sur la périphérie du parc. Or, comme relevé plus haut, la mise sur pied d'un bon plan de gestion nécessite une bonne connaissance du milieu. La question fondamentale de cette recherche s'articule donc autour de la connaissance de la ressource ligneuse dans le PNW dans l'optique d'une meilleure gestion et conservation de ce parc. 
Dans la périphérie du PNW, on a assisté à une diminution de la ressource floristique (Kabelong Banoho, 2013) conséquence des activités anthropiques pratiquées par les populations. En effet, les communautés pauvres en général, ont pour activité lucrative la vente du bois, la culture du sorgho, principales activités responsables de la destruction du couvert ligneux et de la diminution de la faune sauvage (Jiagho, 2009 ; Kabelong Banoho, 2013). Ces activités risquent de compromettre à moyen terme la durabilité des ressources floristiques et fauniques du Parc, car une fois que le stock végétal dans la périphérie du Parc sera menacé, la ressource ligneuse du Parc sera elle aussi entamée. La présence étude avait pour objectif d'améliorer la connaissance de la flore et de la végétation ligneuse afin de permettre de mieux bâtir les stratégies de gestion de ce parc.

\section{MATERIEL ET METHODES}

\section{Localisation et description du site d'étude}

Le Parc National de Waza est situé dans la zone soudano-Sahélienne (Mvondo et al., 2003 ; MINFOF, 2014). Il est encadré par les parallèles $11^{\circ} 03^{\prime}$ et $11^{\circ} 30^{\prime} \mathrm{N}$ et les méridiens $14^{\circ} 20^{\prime}$ et $14^{\circ} 66^{\prime} \mathrm{E}$. Sur le plan administratif, le Parc National de Waza est situé dans la région de l'Extrême-nord Cameroun et plus précisément dans le Département du Logone et Chari (Figure 1). Sur le plan régional, ce Parc est une aire protégée qui se situe à $10 \mathrm{~km}$ à l'Ouest du Nigéria et à $20 \mathrm{~km}$ à l'Est du Tchad.

Le PNW est une aire protégée de catégorie II, selon la classification de l'UICN. D'après le Ministère des Forêts et de la Faune (MINFOF), c'est une aire protégée de Catégorie II et classée Unité Technique Opérationnelle (UTO) de catégorie I de par sa superficie. Le PNW représente à lui seul $90 \%$ des aires protégées de la Région de l'Extrêmenord. Mais ce Parc est sujet à plusieurs types d'occupation et fait l'objet de nombreuses pressions d'origine anthropique. Sa position, son écosystème (plaine inondable), et la population qui vit à sa périphérie fait de Parc l'un des plus exposé aux activités anthropiques. Le dispositif de sondage est présenté dans la Figure 2.

\section{Matériel technique}

Un certain nombre de matériel a permis de mener à bien cette étude. Il est constitué du matériel de collecte et celui du traitement des données. Le matériel de collecte de données est résumé ci-dessous :

- un GPS (Global Positionning System) qui a permis de faire les levées de terrain, de retrouver les débuts des quadrats afin de positionner les quadrats ;

- une boussole qui nous permis d'orienter les quadrats ;

- des rubans de 1,5 $\mathrm{m}$ de long qui ont permis de mesurer la circonférence des arbres ;

- Un décamètre utilisé pour la délimitation des quadrats ;

- des fiches de relevés floristiques pour collecter les données sur le terrain ;

- un appareil photo numérique pour la prise d'image ;

- une perche graduée pour la meure des hauteurs des arbres ;

- un tableau avec les coordonnées géographiques (longitude, latitude) des points d'inventaire ;

- une carte de sondage.

Le matériel de traitement des données est constitué des logiciels de traitement de données: Mapinfo pour la réalisation des cartes, Google Earth pour l'acquisition d'image satellite, Microsoft Office 2010 pour la saisie et le traitement des données.

\section{Méthodes}

Pour mener à bien cette étude, la méthode de collecte, d'analyse et de traitement de données suivante a été retenue.

\section{Collecte des données}

Méthode des quadrats

Dans le cadre de la présente étude, les placettes rectangulaires d'échantillonnage de dimension $25 \mathrm{~m} \times 50 \mathrm{~m}$ (soit $1250 \mathrm{~m}^{2}$ ) et Zapfack (2005) ont été placées sur les transects préalablement définis (Figure 2). Ces placettes (quadrats) ont été positionnées à l'aide d'un GPS sur chaque transect. Les quadrats sont équidistants de $1114 \mathrm{~m}$. Cette approche a permis de parcourir toutes les phytochories du 
Parc. La surface correspondant à chaque quadrat, soit $1250 \mathrm{~m}^{2}$ est subdivisée en de petites bandes de $50 \mathrm{~m} \times 5 \mathrm{~m}$. Tous les individus dont le Diamètre à Hauteur de Poitrine (DBH) et supérieur ou égal à $4 \mathrm{~cm}$ ont été mesurés à l'aide d'un ruban de diamètre, et ceux de diamètre inférieur à $4 \mathrm{~cm}$ ont été signalés. Les espèces ligneuses inventoriées sont marqués pour ne pas être comptés deux fois. La hauteur de chaque individu est mesurée et/ou évaluée. Au total, 500 relevés floristiques (quadrats) ont été réalisés. Cette méthodologie a été adaptée de White et Edwards (2000), Zapfack (2005) et Jiagho et al. (2016).

L'équipe de comptage est constituée de quatre personnes (Picard, 2006) plus un prospecteur principal : un pointeur qui aligne avec le GPS un jalon que tient un jalonneur. Arrivé au début de chaque transect, le pointeur se met sur la ligne de base. Chaque compteur prend une extrémité du décamètre dont la longueur est ajustée à la largeur de la placette d'inventaire $(25 \mathrm{~m})$, la longueur est aussi mesurée soit $50 \mathrm{~m}$. Les limites de la placette sont balisées à l'aide de voyants (branche et feuille peints) et les ligneux contenus dans la placette rectangulaire sont repérés. Les espèces situées à la limite des placettes sont comptées. Une fois les voyants installés, les deux compteurs (prospecteurs) marchent dans les bandes de $5 \mathrm{~m} \quad$ x $50 \mathrm{~m}$ préalablement subdivisées en identifiant en langue locale les espèces, en mesurant la hauteur et la circonférence pour chaque espèce.

\section{Mesure des paramètres dendrométriques}

Pour chaque espèce ligneuse identifiée, les données suivantes sont collectées: la hauteur à l'aide d'une perche graduée et le diamètre à l'aide d'un mètre ruban. Les indices tels que l'état des arbres étaient notés (morts, coupés, défoliés ou entiers) et l'état des branches était signalé (coupées ou non).

La mesure du diamètre des différentes espèces se fait à hauteur de poitrine c'est-à-dire à $1,30 \mathrm{~m}$. Dans le cas de certains arbres, il apparaît que certaines branches se ramifient à une hauteur inférieure à 1,3 m. La mesure du diamètre à hauteur de poitrine revient donc à surestimer le nombre d'arbres. Dans ce cas il convient de mesurer le diamètre à la base, soit
$10 \mathrm{~cm}$ comme préconisé par Henry et al. (2009).

\section{Traitement et analyse des données}

Les fiches d'inventaire ont été dépouillées manuellement puis saisies à partir d'Excel. Les classes de diamètres ont été regroupées en sept modalités d'amplitude vingt centimètre. Ce regroupement a permis d'analyser la structure horizontale du peuplement.

La description de la structure verticale de l'écosystème a été réalisée sur la base des paramètres tels que : la détermination de la proportion des différents types de tiges (tiges de régénération, tiges d'avenir, tiges moyennes, hautes tiges) (Sandjong Sani et al., 2013 ; Jiagho et al., 2016). Parmi les différents types de tiges, compte tenu du fait que l'étude est menée en zone de savane, les tiges de régénération ont été considérées comme celle ayant une taille inférieure ou égale à $1,5 \mathrm{~m}$; les tiges d'avenir celles dont la hauteur est comprise entre $] 1,5 \mathrm{~m} ; 2,5 \mathrm{~m}]$; les tiges moyennes celles situées dans l'intervalle] 2,5 $\mathrm{m} ; 4 \mathrm{~m}]$ et les hautes tiges celles se situant audelà de $4 \mathrm{~m}$.

Taux de sondage

La gestion durable de la ressource ligneuse est une préoccupation majeure pour la stabilité des écosystèmes soudano-sahélien, cette étude se fonde sur un inventaire de ce type de ressource. Il s'est effectué à un taux de sondage $0,04 \%$. Celui-ci a tenu compte de la superficie du PNW qui est de 170000 ha. Pour Bellefontaine (1997), dans les travaux d'aménagement forestier, un échantillonnage préliminaire à très faible taux de sondage est à préconiser, afin de caractériser l'hétérogénéité de la ressource et d'estimer le nombre minimum de placettes nécessaires pour atteindre une précision donnée. Dans le cadre de cette étude, 500 placettes de $1250 \mathrm{~m}^{2}$ (25 m x $50 \mathrm{~m}$ ) ont été inventoriées.

\section{Densité absolue}

La densité absolue indique la valeur moyenne du nombre d'individus de l'espèce par unité d'échantillon. Dans le cadre de cette recherche, elle a été calculée par hectare. Selon 
la formule (Jiagho et al., 2016 ; Mbaiyetom et al., 2021).

$$
D a=\frac{\text { ni }}{\mathrm{M}}
$$

Avec ; Da : Densité absolue ;

ni : nombre d'individus de l'espèce ;

M: Superficie totale des unités échantillonnées.

\section{Diversité générique}

Une flore est diversifiée lorsqu'elle renferme moins de grands genres plurispécifiques (Kabelong Banoho, 2013; Jiagho et al., 2016). Le coefficient générique est le rapport entre le nombre de genres et celui des espèces.

$$
C G=\frac{\text { Nombre de genre }}{\text { Nombre des espèces }} \times 100
$$

Indice de Shannon-Weaver

La diversité Alpha permet d'évaluer le poids de l'espèce dans l'occupation du sol en utilisant l'indice de diversité de Shannon-
Weaver. Cet indice varie en fonction du nombre d'espèces présentes. Il est d'autant plus élevé qu'un grand nombre d'espèces participe dans l'occupation du sol. Il s'exprime en bits par individu. (Sandjong Sani et al., 2013; Honvou et al., 2021).

$$
H^{\prime}=\sum_{i=0}^{n} P_{i} \log _{2} P_{i}
$$

Avec: Pi: la proportion relative du recouvrement moyen de l'espèce $\mathrm{i}$ (valeurs comprises entre 0 et 1 );

$\log _{2}$ : le logarithme à base 2 .

Equitabilité de Piélou

$$
E=\frac{H^{\prime}}{\log _{2} S}
$$

Elle tend vers 0 lorsqu'il y a dominance et vers 1 lorsqu'un maximum d'espèces participe au recouvrement (Sandjong Sani et al., 2013 ; Honvou et al., 2021).

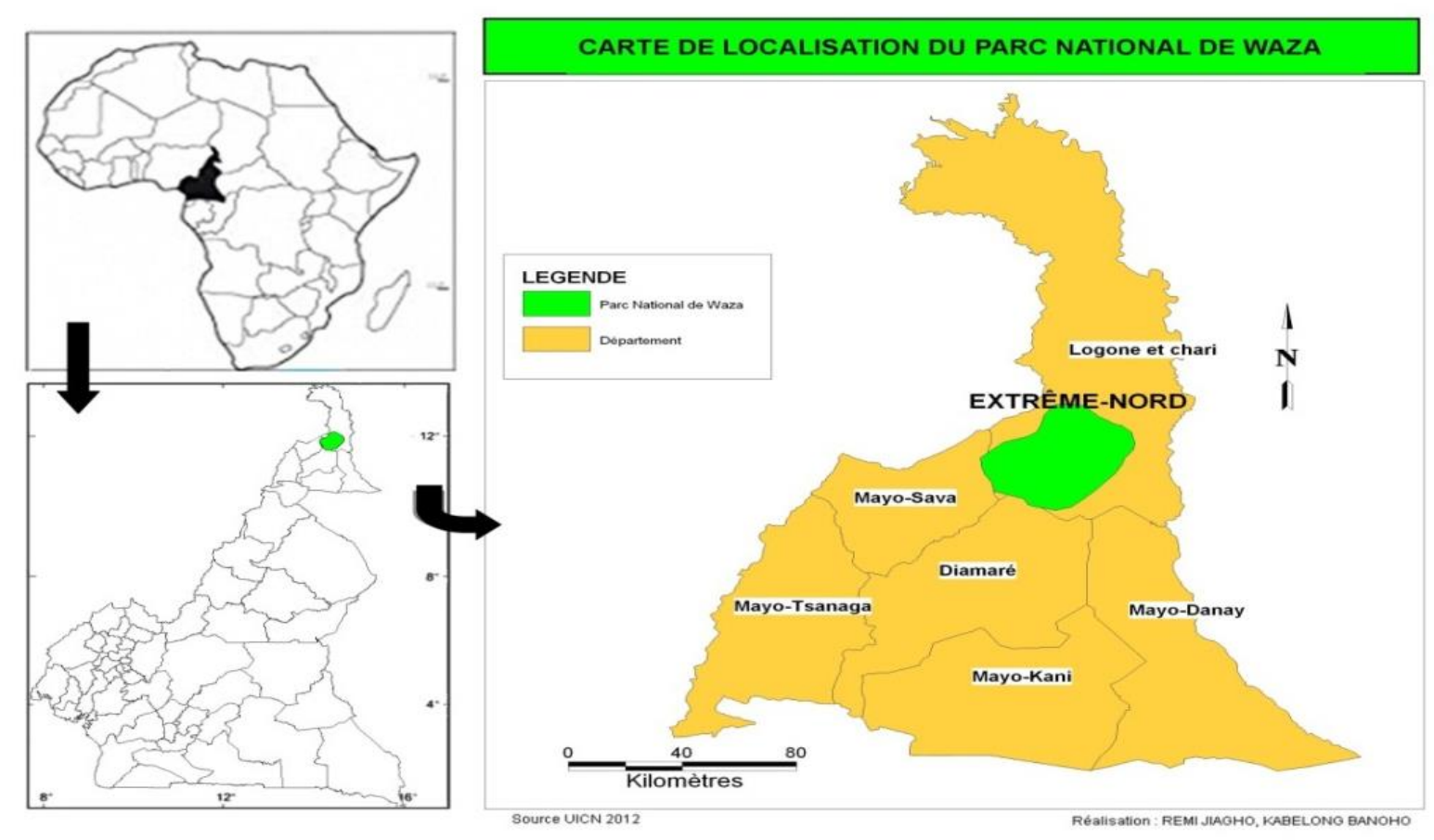

Figure 1: Localisation de la zone d'étude. 


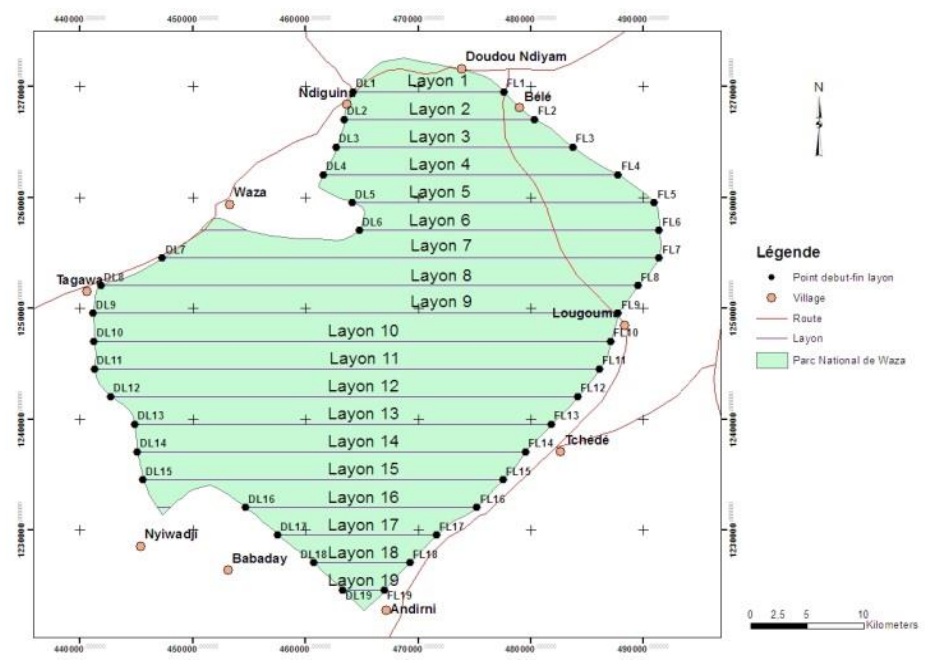

Figure 2: Dispositif de sondage de la zone d'étude.

\section{RESULTATS}

\section{Sol et physionomie de la végétation}

L'appréciation visuelle des différentes strates dans toutes les placettes donne une physionomie de forêts sèches claires à denses dans la partie Ouest du Parc d'où le nom «Zone forestière» avec parfois, une végétation ligneuse importante, à couvert plus ou moins fermé, et de multiples individus à hauteur supérieure à $5 \mathrm{~m}$. Dans la partie centrale par contre, on note la dominance d'une savane arbustive avec une dominance des espèces telles que Acacia seyal. Le tapis graminéen associé à d'autres herbacées observées dans la zone Nord-Est est caractéristique de la zone inondable localement appelé «Yaérés».

Suivant la texture du sol, on peut diviser le parc en deux grandes régions naturelles bien distinctes suivant les conditions topographiques et le régime hydrique annuel auquel elles sont soumises :

- la partie centrale et orientale du parc, dont la côte varie entre 299 et 305 m, est inondée pendant une plus ou moins longue période de l'année

- la partie occidentale du parc, située à une côte un peu plus élevée oscillant entre 305 et $311 \mathrm{~m}$, est très peu inondée. Mais l'on retrouve de grandes mares pendant la saison de pluie du fait de la qualité du sol qui s'y trouve.

Mais suivant les types de végétation associés, on distingue 03 types de paysage. Il s'agit de :

- La plaine inondable, située dans la partie Centre-Est et Nord-Est du parc. Elle constitue un biotope très particulier entièrement développé sur des sédiments fins argilo limoneux, où le minéral argileux dominant est la montmorillonite (UICN/CBLT, 2007b). C'est le domaine des vertisols, bien connus localement sous les noms de Karal au Nord-Cameroun. Cette partie du Parc est dominée par des Graminées et est parsemée de quelques Acacia seyal et Calotropis procera.

- La savane à Combretaceae sur sols ferrugineux tropicaux dans la partie SudOuest du Parc. Cette formation, qui occupe la partie topographiquement la plus élevée du parc, se développe sur un matériau homogène très sableux, d'origine dunaire, dans lequel se différencient les sols ferrugineux tropicaux lessivés. Cette partie est dominée par des espèces telles que Guiera senegalensis, Combretum molle et Anogeissus leiocarpus.

- Les formations végétales sur sols lessivés et alcalins. Elles se développent le long de la bordure sud-occidentale du parc, sur une 
dorsale très peu marquée grossièrement orientée nord-sud, à texture d'ensemble argileuse. Cette partie est marquée par une savane arbustive dominée par Acacia seyal. On la localise dans la partie Nord-Ouest et la partie centrale.

\section{Richesse floristique}

L'analyse de la richesse floristique a permis d'identifier 38 espèces dans l'ensemble des placettes. Le Tableau 1 donne la liste des espèces végétales, les noms vernaculaires, les familles correspondantes. La flore du parc est donc riche de 38 espèces appartenant à 33 genres répartis dans 19 familles.

Sur les 6752 individus inventoriés, la famille des Fabaceae est la plus abondante, avec 3441 individus recensés. Elle est suivie respectivement des familles de Combretaceae (2049 individus), Balanitaceae (468 individus), Anacardiaceae (248 individus), Arecaceae (186 individus), Rhamnaceae (122 individus), Meliaceae (73 individus), Bignoniaceae (59 individus), Annonaceae (29 individus), Rubiaceae (28 individus). Les familles les moins représentées en individus ou rares sont les suivantes : Sterculiaceae (15 individus), Asclepiadaceae (14 individus), Loganiaceae (06 individus), Capparaceae (06 individus), Ulmaceae (03 individus), Tiliaceae (02 individus), Bombacaceae (01 individu), Ebenaceae (01 individu), Verbenaceae (01 individu).

\section{Richesse spécifique et densité absolue}

Le Tableau 2 donne des informations sur la qualité et la quantité de la ressource ligneuse présente dans le PNW. Il montre que le nombre moyen de tige à l'hectare est de 108,03 individus/ha, le coefficient de variation (CV rapport en pourcentage entre l'écart type des mesures et la moyenne) est de $294,1 \%$, ce CV représente la dispersion autour de la moyenne. Le calcul de l'erreur avec un seuil de probabilité de 0,5 a donné $26,3 \%$, ce qui a permis de conclure que, sur l'ensemble du site, le nombre moyen de tiges par hectare a $95 \%$ de chances de se trouver entre 79,6 et 136,44 tiges/ha.

\section{Structure diamétrique}

L'analyse des données des classes de diamètre montre que la majorité des individus se retrouve dans la classe [0-20 cm[. Cette classe représente à elle seule $71 \%$ des individus recensés. Pour les autres classes, les effectifs sont repartis de manière suivante : $26,48 \%$ pour la classe $[20-40 \mathrm{~cm}[$, de $2,1 \%$ pour la classe [ $40-60 \mathrm{~cm}$ [ et $0,46 \%$ pour toutes les classes de diamètre supérieur ou égal à 60 $\mathrm{cm}$ (Figure 3). Les espèces sont essentiellement de petit diamètre à hauteur de poitrine.

\section{Diversité et équitabilité floristiques}

Les indices de diversité calculée illustrent l'état de l'écosystème (Tableau 3). L'indice de diversité de Shannon-Weaver ( $\mathrm{H}$ ') calculés est de 2, 95. Il traduit une diversité floristique moyenne du PNW. Le calcul de l'équitabilité de Piélou $(\mathrm{E}=0,56)$ rattachée à l'indice de Shannon confirme les valeurs d'indices de diversité moyenne sachant que les valeurs qui se rapprochent de l'unité ont une diversité spécifique grande. Il y a donc $56 \%$ de chance qu'il y ait équilibre dans la répartition des espèces dans le parc. La diversité générique est élevée du fait du grand nombre de genre.

En faisant une analyse avec le Tableau 4 proposé par Simboura et Zenetos (2002), on pourrait conclure que l'état de l'écosystème est médiocre donc il est fortement dégradé. Pour une zone de forêt tropicale humide, cette affirmation devrait être valide. Compte tenu des conditions biologiques et climatiques des zones de savane sèche, cette affirmation ne trouverait pas de fondement.

\section{Structure verticale et type végétatif}

L'analyse de la structure verticale montre une distribution plus ou moins équilibrée pour les différents types de tiges. $\mathrm{La}$ Figure 4 montre que 21,3\% sont des tiges de régénération, $16,5 \%$ sont des tiges d'avenir, $28,7 \%$ sont des tiges moyennes et $33,4 \%$ sont de hautes tiges, ce qui traduit l'aspect savane arborescent observé dans la partie Ouest du Parc.

La Figure 5 montre la répartition le nombre d'individus appartenant à chaque type végétatif. Il en ressort que $66,59 \%$ des 
individus sont des arbustes, tandis que $33,41 \%$ sont des arbres.

\section{Dominance et surface terrières des espèces}

Plusieurs espèces se distinguent par leur effectif élevé. Les espèces assez représentatives pour caractériser la végétation du parc sont : Acacia seyal (46,52\%), Guiera senegalensis $(11,42 \%)$, Combretum molle (7,48\%), Balanites aegyptiaca $(6,93 \%) C$. aculeatum (5,47\%), Terminalia laxiflora (4,74\%) Hyphaena thebaica (2,75\%), Piliostigma reticulatum (2,38\%), Lannea humilis (2,25\%), Anogeissus leiocarpus (1,24\%), Acacia hockii (1,14\%) et Azadirachta indica $(1,07 \%)$.

Plusieurs espèces peuvent être considérées comme accidentelles ou rares, car elles sont représentées par un seul individu : Diospiros mespiliformis, Adansonia digitata, Acacia ataxancantha, Khaya senegalensis et Vitex doniana.

D'autres espèces sont représentées très faiblement, parce que liées à la présence humaine ou à leur probable introduction par l'avifaune ou les Chiroptères ou dû simplement au taux de sondage qui est de $0,04 \%$. Il s'agit de : Pteurocarpus lucens, Entada africana, Gardenia aqualla, Grewia molis et Faidherbia africana.
Le Tableau 5 montre que Acacia seyal est l'espèce ayant une grande surface terrière avec 14,6 $\mathrm{m}^{2} / \mathrm{ha}$, suivit de Balanites aegyptiaca (3,9 $\left.\mathrm{m}^{2} / \mathrm{ha}\right)$, Anogeissus leiocarpus $(2,1 \mathrm{~m} / \mathrm{ha})$, Sclerocarya birrea $\left(1,7 \mathrm{~m}^{2} / \mathrm{ha}\right)$ et Combretum molle $\left(1,5 \mathrm{~m}^{2} / \mathrm{ha}\right)$. Les espèces ayant une faible surface terrière sont Acacia ataxancantha $\left(0,001 \mathrm{~m}^{2} / \mathrm{ha}\right)$ suivit de Grewia molis $(0,0007$ $\left.\mathrm{m}^{2} / \mathrm{ha}\right)$, Diospiros mespiliformis $(0,0006$ $\left.\mathrm{m}^{2} / \mathrm{ha}\right)$ et Vitex doniana $\left(0,0004 \mathrm{~m}^{2} / \mathrm{ha}\right)$.

\section{Perturbation de la ressource}

Pendant la phase de collecte de données, des indices de présence humaine ont été observés. Les coupes de bois, les pistes de braconniers, les prélèvements de pailles sont observés dans les placettes. La Figure 6 illustre que les ligneux effeuillés, coupés à la tige et coiffés sont les plus nombreux avec respectivement 317, 200 et 176 individus. Les espèces les plus convoitées par ordre d'importance pour les différentes activités anthropiques (branches coupées en têtard ou émondées) sont : Acacia seyal, Balanites aegyptiaca, Combretum molle, Piliostigma reticulatum, Sclerocarya birrea, Stereospermum kunthianum et Terminalia laxiflora.

Tableau 1: Familles, noms scientifiques et noms en langues locales.

\begin{tabular}{lll}
\hline Familles & Noms Scientifique & Noms en langue locale \\
\hline Anacardiaceae & Lannnea humilis (Oliv.) Engl. & Belouki (Fu) \\
& Sclerocarya birrea (A. Rich.) Hochst. & Eri (Fu) \\
Annonaceae & Annona senegalensis Pers. & Dukouhi (Fu) \\
Asclepiadaceae & Calotropis procera (Ait.) Ait. & Babambi (Fu) \\
Arecaceae & Hyphaena thebaica (Linn.) Mart. & Gellehi (Fu) \\
Balanitaceae & Balanites aeggyptiaca (Linn.) Del. & Tanné (Fu) \\
Bignoniaceae & Stereospermum kunthianum Cham. & Golombi (Fu) \\
Bombacaceae & Adansonia digitata Linn & Bokki \\
Capparaceae & Boscia senegalensis (Pers.) Lam. & Tambila (Md) \\
Combretaceae & Anogeissus leiocarpus (DC.) Guill. et Perr. & Kodjoli (Fu) \\
& Combretum aculeatum Vent. & Gourdiak (Mf) \\
& C. molle R. Br. ex G. Don & Dodji (Fu) \\
\hline
\end{tabular}




\begin{tabular}{|c|c|c|}
\hline & Guiera senegalensis Lam. & Guelehouki (Fu) \\
\hline & Terminalia laxiflora Engl. et Diels & Koulahi (Fu) \\
\hline \multirow[t]{6}{*}{ Ebenaceae } & Diospiros mespiliformis Hochst. ex A. DC. & Nelbi \\
\hline & Acacia hockii De wild & Tchilouki (Fu) \\
\hline & A. nilotica Wild. & Gabdi/Gawari (Fu) \\
\hline & A. senegal (L.) Wild. & Patuki/Patugelhi (Fu) \\
\hline & A. seyal Del. & Boulbi (Fu) \\
\hline & Dalbergia melanoxylon Guill. et Perr. & Ngalalahi (Fu) \\
\hline \multirow[t]{7}{*}{ Fabaceae } & Dichrostachys cinerea (L.) Wight et Arn. & Burli (Fu) \\
\hline & Entada africana Guill. et Perr. & Fadowanduhi $(\mathrm{Fu})$ \\
\hline & Faidherbia albida Del. & Tchaski (Fu) \\
\hline & A. ataxacantha $D C$. & Zaza (Md) \\
\hline & Piliostigma reticulatum (DC.) Hochst. & Barkehi (Fu) \\
\hline & Pterocarpus lucens Lepr. ex Gui. et Per. & Tiami (Fu) \\
\hline & Tamarindus indica Linn. & Djabbé (Fu) \\
\hline Loganiaceae & Strychnos spinosa Lam. & Tumokohi \\
\hline \multirow[t]{2}{*}{ Meliaceae } & Azadirachta indica A. Juss. & Gagné (Fu) \\
\hline & Khaya senegalensis (Desr.) A.Juss. & Serehi (Fu) \\
\hline \multirow[t]{2}{*}{ Rhamnaceae } & Ziziphus mauritiana Lam. & Djabi (Fu) \\
\hline & Z. spina-christi (Linn.) Desf. & Kurnahi (Fu) \\
\hline \multirow[t]{2}{*}{ Rubiaceae } & Gardenia aqualla Stapf et Hutch. & \\
\hline & Mitragyna inermis O. Kuntze & Koli (Fu) \\
\hline Sterculiaceae & Sterculia setigera Del. & ZhawaZehe (Md) \\
\hline Tiliaceae & Grewia mollis Juss. & Kelli (Fu) \\
\hline Ulmaceae & Celis integrifolia Lam. & Ganki (Fu) \\
\hline Verbenaceae & Vitex doniana Sweet & Galbihi (Fu) \\
\hline
\end{tabular}

Tableau 2 : Espèces, DBH et densité absolue.

\begin{tabular}{lcccccccc}
\hline Noms scientifiques & {$[\mathbf{0 - 2 0}[$} & {$[\mathbf{2 0 - 4 0}[$} & {$[\mathbf{4 0 - 6 0}[$} & $\mathbf{6 0 - 8 0}[$ & $\mathbf{6 0 - 1 0 0}[$ & $\mathbf{1} \mathbf{1 0 0}$ & Effectifs & Da \\
\hline Acacia ataxancatha & 1 & 0 & 0 & 0 & 0 & 0 & 1 & 0,02 \\
A. hockii & 17 & 49 & 10 & 1 & 0 & 0 & 77 & 1,23 \\
A. nilotica & 4 & 0 & 0 & 0 & 0 & 0 & 4 & 0,06 \\
A. senegal & 15 & 5 & 0 & 0 & 0 & 0 & 20 & 0,32 \\
A. seyal & 1891 & 1225 & 25 & 0 & 0 & 0 & 3141 & 50,26 \\
Adansonia digitata & 0 & 0 & 1 & 0 & 0 & 0 & 1 & 0,02 \\
Annona senegalensis & 29 & 0 & 0 & 0 & 0 & 0 & 29 & 0,46 \\
Anogeissus leiocarpus & 14 & 34 & 24 & 10 & 1 & 1 & 84 & 1,34 \\
Azadirachta indica & 15 & 53 & 3 & 1 & 0 & 0 & 72 & 1,15 \\
Balanites aegyptiaca & 181 & 254 & 26 & 6 & 1 & 0 & 468 & 7,49 \\
Boscia senegalensis & 6 & 0 & 0 & 0 & 0 & 0 & 6 & 0,10 \\
\hline
\end{tabular}




\begin{tabular}{|c|c|c|c|c|c|c|c|c|}
\hline Calotropis procera & 13 & 1 & 0 & 0 & 0 & 0 & 14 & 0,22 \\
\hline Celtis integrifolia & 1 & 0 & 2 & 0 & 0 & 0 & 3 & 0,05 \\
\hline Combretum aculeatum & 369 & 0 & 0 & 0 & 0 & 0 & 369 & 5,90 \\
\hline C. molle & 434 & 63 & 8 & 0 & 0 & 0 & 505 & 8,08 \\
\hline Dalbergia melanoxylon & 10 & 0 & 0 & 0 & 0 & 0 & 10 & 0,16 \\
\hline Dichrostachys cinerea & 6 & 0 & 0 & 0 & 0 & 0 & 6 & 0,10 \\
\hline Diospiros mespiliformis & 1 & 0 & 0 & 0 & 0 & 0 & 1 & 0,02 \\
\hline Entada africana & 3 & 0 & 0 & 0 & 0 & 0 & 3 & 0,05 \\
\hline Faidherbia albida & 1 & 1 & 0 & 0 & 0 & 0 & 2 & 0,03 \\
\hline Gardenia aqualla & 2 & 0 & 0 & 0 & 0 & 0 & 2 & 0,03 \\
\hline Grewia mollis & 2 & 0 & 0 & 0 & 0 & 0 & 2 & 0,03 \\
\hline Guiera senegalensis & 767 & 4 & 0 & 0 & 0 & 0 & 771 & 12,34 \\
\hline Hyphaena thebaica & 168 & 18 & 0 & 0 & 0 & 0 & 186 & 2,98 \\
\hline Khaya senegalensis & 1 & 0 & 0 & 0 & 0 & 0 & 1 & 0,02 \\
\hline Lannea humilis & 152 & 0 & 0 & 0 & 0 & 0 & 152 & 2,43 \\
\hline Mitragyna inermis & 23 & 3 & 0 & 0 & 0 & 0 & 26 & 0,42 \\
\hline Piliostigma reticulatum & 153 & 8 & 0 & 0 & 0 & 0 & 161 & 2,58 \\
\hline Pterocarpus lucens & 4 & 0 & 0 & 0 & 0 & 0 & 4 & 0,06 \\
\hline Sclerocarya birrea & 23 & 37 & 29 & 7 & 0 & 0 & 96 & 1,54 \\
\hline Sterculia setigera & 15 & 0 & 0 & 0 & 0 & 0 & 15 & 0,24 \\
\hline \multicolumn{9}{|l|}{ Stereospermum } \\
\hline kunthianum & 36 & 14 & 8 & 0 & 1 & 0 & 59 & 0,94 \\
\hline Strychnos spinosa & 6 & 0 & 0 & 0 & 0 & 0 & 6 & 0,10 \\
\hline Tamarindus indica & 2 & 6 & 2 & 1 & 1 & 0 & 12 & 0,19 \\
\hline Terminalia laxiflora & 308 & 11 & 1 & 0 & 0 & 0 & 320 & 5,12 \\
\hline Vitex doniana & 1 & 0 & 0 & 0 & 0 & 0 & 1 & 0,02 \\
\hline Ziziphus mauritiana & 117 & 1 & 0 & 0 & 0 & 0 & 118 & 1,89 \\
\hline Z. spina-christi & 3 & 1 & 0 & 0 & 0 & 0 & 4 & 0,06 \\
\hline Total & 4794 & 1788 & 139 & 26 & 4 & 1 & 6752 & 108,03 \\
\hline
\end{tabular}

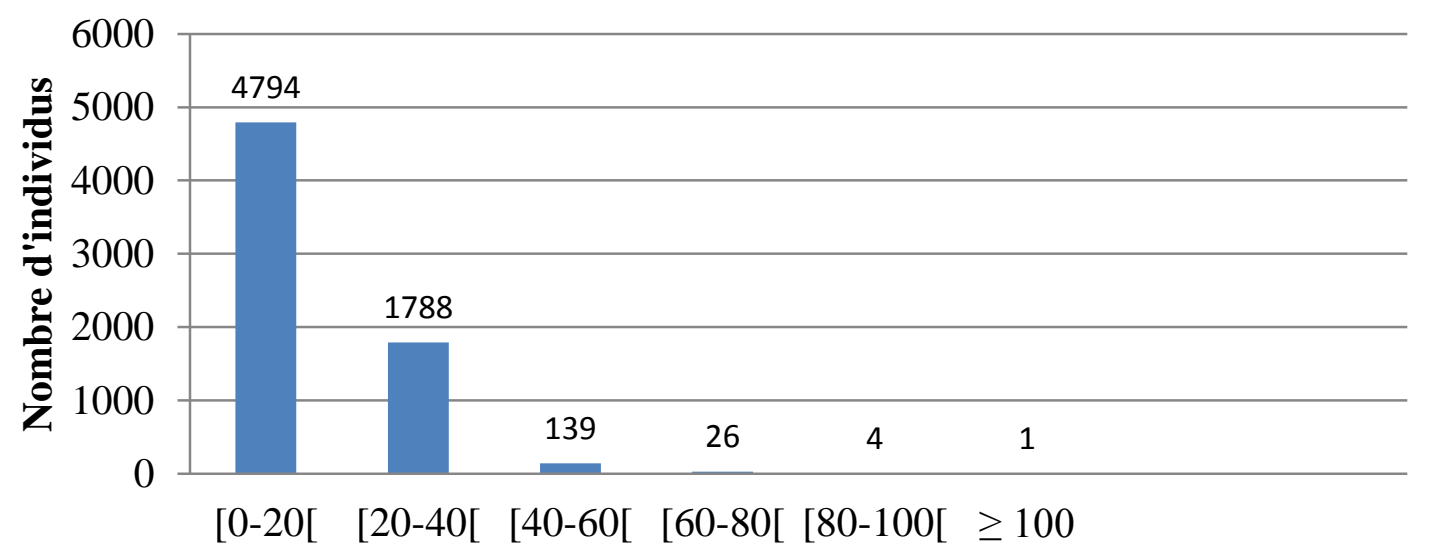

Classes de diamètre

Figure 3: Fréquence de distribution par classe de diamètre. 
Tableau 3 : Récapitulatif des indices et leur valeur.

\begin{tabular}{lc}
\hline Indices & Valeurs \\
\hline Densité absolue (Da) & 108,03 individus/ha \\
Indice de Shannon Weaver (H') & 2,95 bits \\
Equitabilité de Piélou & 0,56 \\
Diversité générique (CG) & 86,84 \\
\hline
\end{tabular}

Tableau 4 : Etat de l'écosystème et variation de l'indice de Shannon.

\begin{tabular}{lcc}
\hline Etat & Variation Indice de Shannon & Commentaire \\
\hline Mauvais & $0 \leq \mathrm{H}^{\prime} \leq 1,5$ & Très dégradé \\
Médiocre & $1,5 \leq \mathrm{H}^{\prime} \leq 3$ & Fortement dégradé \\
Moyen & $3 \leq \mathrm{H}^{\prime} \leq 4$ & Modérément dégradé \\
Bon & $4 \leq \mathrm{H}^{\prime} \leq 5$ & Zone de transition \\
Très bon & $>5$ & Site de référence \\
\hline
\end{tabular}

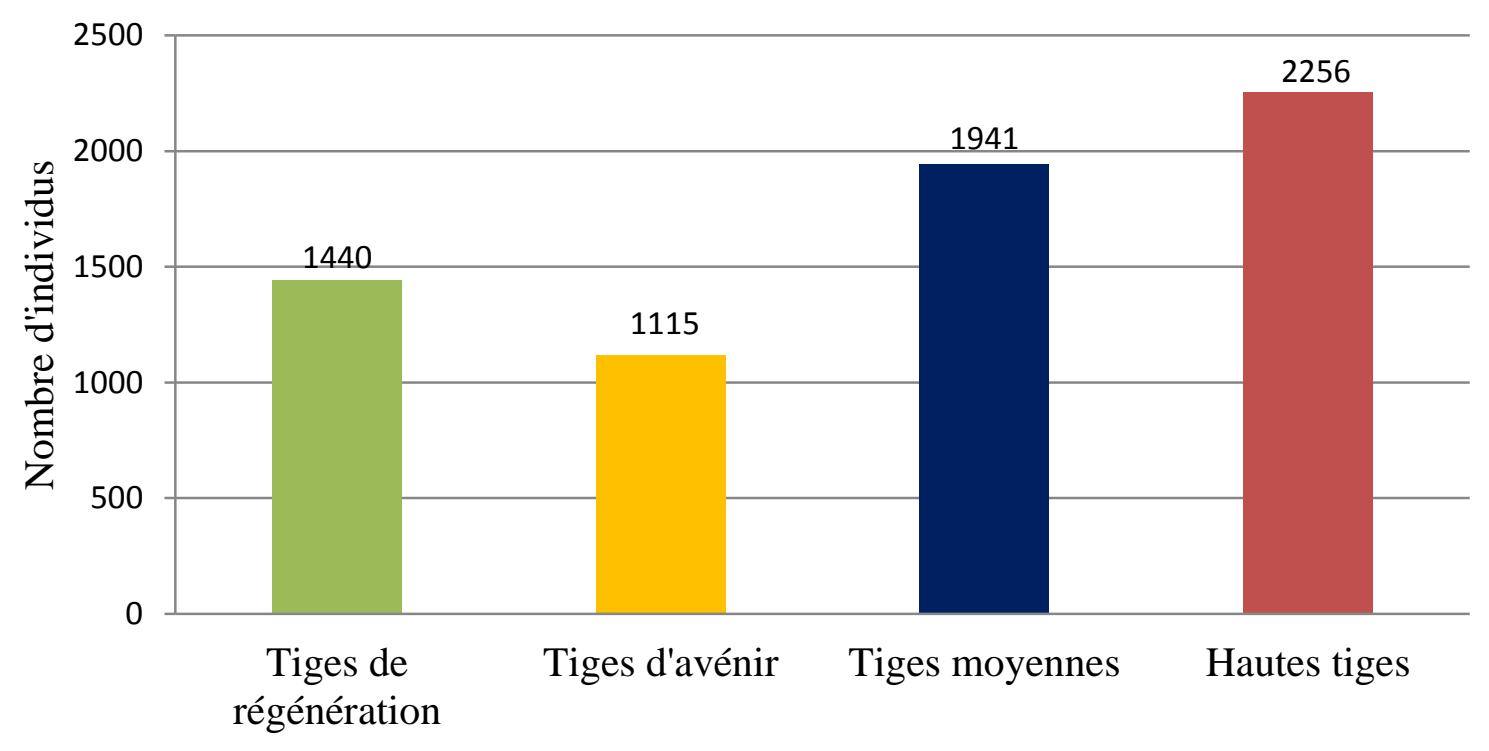

Figure 4: Abondance des différents types de tiges. 


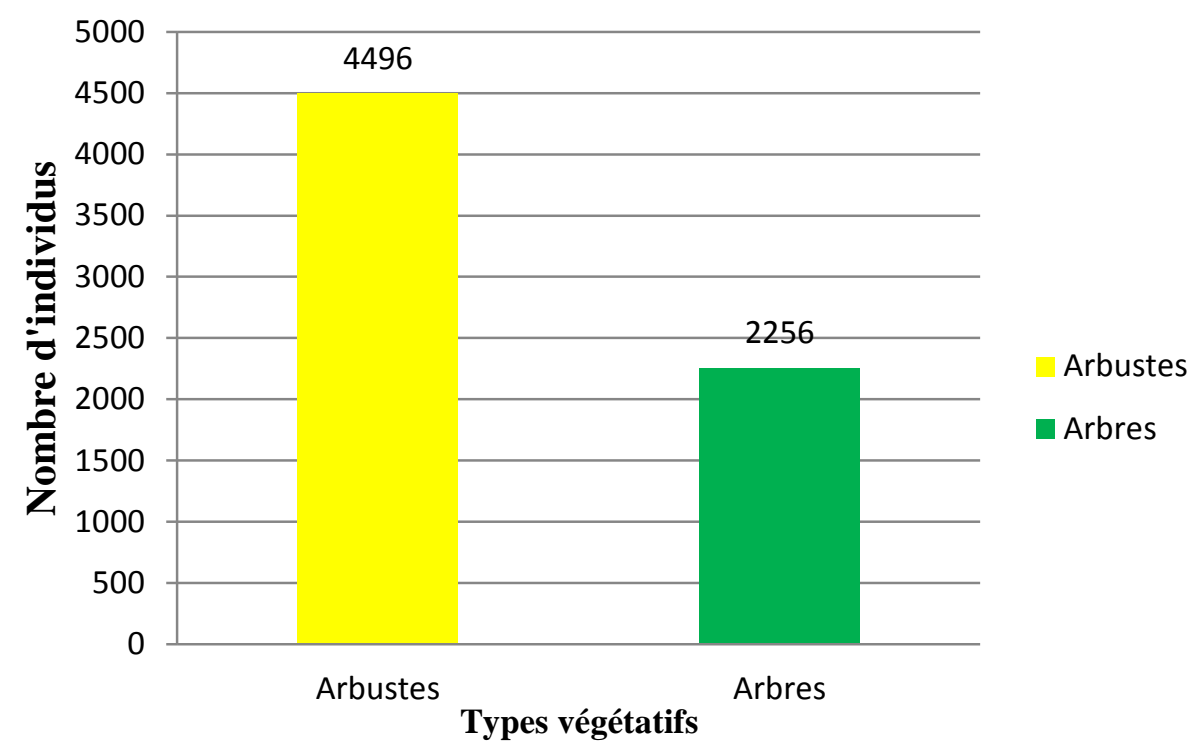

Figure 5: Représentativité des types végétatifs de ligneux.

Tableau 5 : Dominance et surface terrières des espèces.

\begin{tabular}{|c|c|c|c|}
\hline Noms scientifiques & Dominance relative & Dominance absolue & Surface Terrière \\
\hline Acacia seyal & 0,47 & 46,52 & 14,6 \\
\hline Balanites aegyptiaca & 0,07 & 6,93 & 3,9 \\
\hline Anogeissus leiocarpus & 0,01 & 1,24 & 2,1 \\
\hline Sclerocarya birrea & 0,01 & 1,42 & 1,7 \\
\hline Combretum molle & 0,07 & 7,48 & 1,5 \\
\hline A. hockii & 0,01 & 1,14 & 0,8 \\
\hline Azadirachta indica & 0,01 & 1,07 & 0,7 \\
\hline $\begin{array}{l}\text { Stereospermum } \\
\text { kunthianum }\end{array}$ & 0,01 & 0,87 & 0,53 \\
\hline Terminalia laxiflora & 0,05 & 4,74 & 0,5 \\
\hline Guiera senegalensis & 0,11 & 11,42 & 0,43 \\
\hline Piliostigma reticulatum & 0,02 & 2,38 & 0,3 \\
\hline Tamarindus indica & 0,00 & 0,18 & 0,29 \\
\hline Hyphaena thebaica & 0,03 & 2,75 & 0,25 \\
\hline Lannea humilis & 0,02 & 2,25 & 0,14 \\
\hline C. aculeatum & 0,05 & 5,47 & 0,12 \\
\hline Celtis integrifolia & 0,00 & 0,04 & 0,074 \\
\hline A. senegal & 0,00 & 0,30 & 0,07 \\
\hline Mitragyna inermis & 0,00 & 0,39 & 0,07 \\
\hline Annona senegalensis & 0,00 & 0,43 & 0,04 \\
\hline Ziziphus mauritiana & 0,02 & 1,75 & 0,04 \\
\hline
\end{tabular}




\begin{tabular}{lccc}
\hline Calotropis procera & 0,00 & 0,21 & 0,021 \\
Adansonia digitata & 0,00 & 0,01 & 0,02 \\
Khaya senegalensis & 0,00 & 0,22 & 0,02 \\
Dichrostachys cinerea & 0,00 & 0,09 & 0,013 \\
Z. spina-christi & 0,00 & 0,06 & 0,012 \\
Faidherbia albida & 0,00 & 0,03 & 0,01 \\
A. nilotica & 0,00 & 0,06 & 0,008 \\
Dalbergia melanoxylon & 0,00 & 0,15 & 0,008 \\
Gardenia aqualla & 0,00 & 0,03 & 0,006 \\
Strychnos spinosa & 0,00 & 0,09 & 0,006 \\
Pterocarpus lucens & 0,00 & 0,06 & 0,005 \\
Sterculia setigera & 0,00 & 0,01 & 0,004 \\
Boscia senegalensis & 0,00 & 0,09 & 0,003 \\
Entada africana & 0,00 & 0,04 & 0,003 \\
A. ataxancatha & 0,00 & 0,01 & 0,001 \\
Grewia mollis & 0,00 & 0,03 & 0,0007 \\
Diospiros mespiliformis & 0,00 & 0,01 & 0,0006 \\
Vitex doniana & 0,00 & 0,01 & 0,0004 \\
Total & 1 & 100,00 & 28,2957 \\
\hline
\end{tabular}

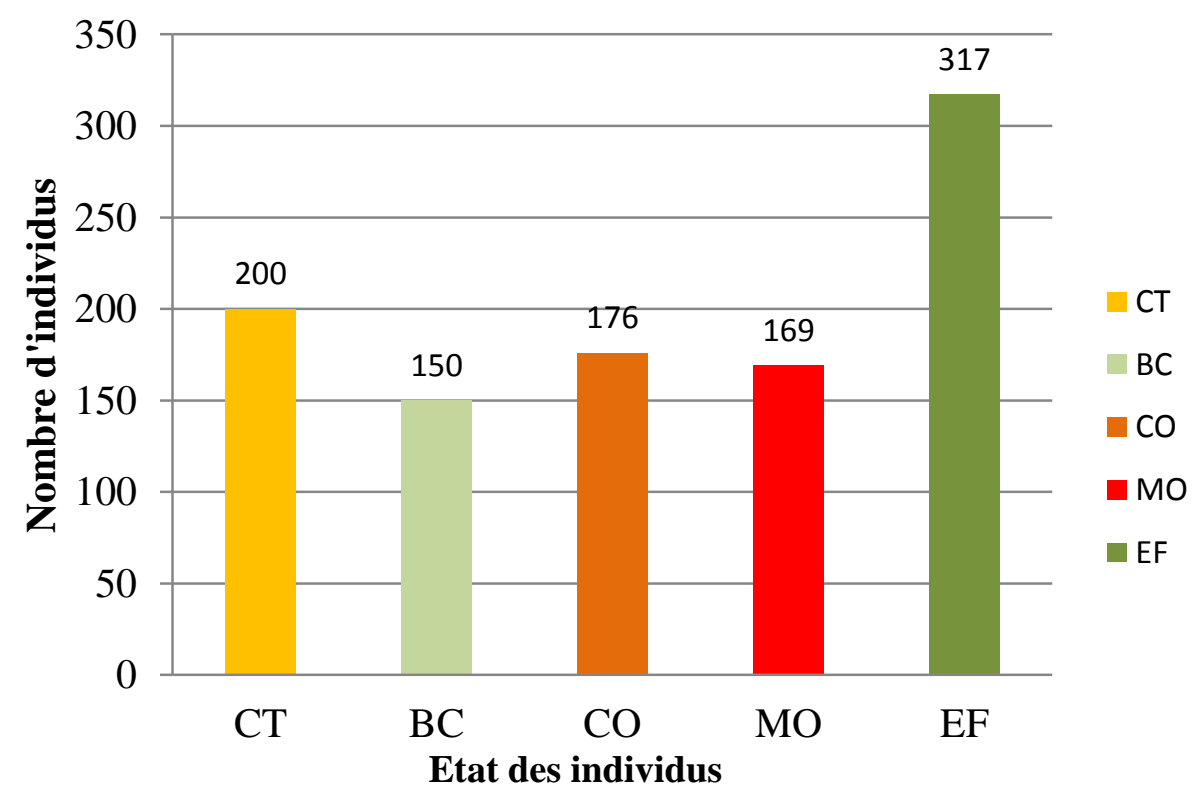

Figure 6: Etat des individus recensés.

$\mathrm{CT}=$ Coupés à la tige $; \mathrm{BC}=$ Branches coupées $; \mathrm{CO}=$ Tiges coiffées ; $\mathrm{MO}=$ Morts ; EF=Effeuillés. 


\section{DISCUSSION}

\section{Méthodologie et pédologie}

L'approche méthodologique adoptée lors de cette étude est celle des quadrats de 25 m x $50 \mathrm{~m}$. Cette méthode est adaptée de celle de Zapfack (2005), Jiagho et al. (2016) et Cisse et al. (2020), qui est relativement plus flexible et moins contraignante. L'échantillonnage est utilisé pour des raisons économiques dans la plupart des inventaires car les écosystèmes boisés sont généralement trop vastes pour être inventoriées « en plein ». L'échantillonnage fournit des estimations de ce que l'on cherche à quantifier à partir d'un nombre limité de mesures prises selon des critères précis (FFEM, 2007). Le taux de sondage est de $0,04 \%$. La relative hétérogénéité entre les placettes montre qu'il faut envisager un inventaire avec un taux de sondage plus grand pour obtenir des caractéristiques plus précises du parc. Toutefois, selon Marcon (2011), l'augmentation de la surface d'échantillonnage n'est pas toujours une solution adaptée en zone tropicale, puisqu'elle se solde toujours par l'augmentation du nombre d'espèces.

La prépondérance de la texture sablolimoneuse dans l'horizon superficiel, l'absence d'érosion et l'activité biologique présentes dans le sol (fourmilières, trous des animaux, etc.) sont des caractéristiques pédologiques favorables à l'épanouissement de la végétation car, la capacité de rétention d'eau et d'air est relativement élevée, l’infiltration, la circulation de l'eau et la minéralisation facilitées. Mais cette analyse laisserait voir un écosystème plus riche, or le nombre d'espèce est plus faible. Ceci se justifie aussi par la grande surface inondable pendant une bonne partie de l'année, qui favorise la croissance des herbacées et défavorise la croissance des ligneux. D'après Gnoumou et al. (2020), les sols à fort taux d'argile imbibés en surface sont particulièrement imperméables et cela limite la progression des racines des jeunes plants ligneux en profondeur; en effet, la présence d'une abondante quantité d'eau sur ces sols entraine leur engorgement qui peut asphyxier également un bon nombre d'espèces dès la germination.

\section{Richesse et diversité floristique}

L'inventaire floristique réalisé dans le cadre de cette étude montre qu'il existe 38 espèces ligneuses, reparties en 33 genres et appartenant à 19 familles dans le PNW. Le nombre d'espèces recensées dans cette étude est plus faible que celui obtenu par Sandjong Sani et al. (2013), qui a trouvé 62 espèces dans le Parc National de Mozogo-Gokoro (Cameroun) et de Jiagho et al. (2016) qui a recensé 52 espèces à la périphérie du PNW. La différence avec le Parc National de Mozogo Gokoro est due au fait que ce parc situé plus du sud et est moins soumis aux facteurs naturels de dégradation. En revanche, la différence observée avec les résultats de la zone périphérique peut s'expliquer tout d'abord par le taux de sondage qui était plus élevé $(0,8 \%)$ et aurait permis de recenser un plus grand nombre d'espèces. Ensuite, l'autre explication relève du fait que, les populations installées à la zone périphérique du parc ont tendance à mieux protéger les rares individus restant des espèces ligneuses menacées de disparition dans les terroirs villageois et les jardins de case compte tenu de leur importance socioéconomique, ce qui a pour avantage une plus grande richesse spécifique à la périphérie du parc.

Mbaiyetom et al. (2021) quant à lui a recensé un nombre d'espèces plus faibles (29 espèces recensées) dans les parcs arborés du Département de la Nya (Sud du Tchad) situés dans la même zone agro-écologique que le PNW. Cette faible valeur par rapport à celle de cette étude est due au fait que ces parcs arborés ne bénéficient d'un statut clair de protection. D'autre part, les moyens de contrôle et de surveillance des gardes forestiers sont très limités sinon inexistant, ce qui favorisent les activités d'exploitation illégales de ligneux qui 
entrainent une forte dégradation de ces ressources.

L'indice de Shannon Weaver obtenu lors de cette étude est de 2,95 bits, l'équitabilité de Piélou de 0,56, la diversité générique de $86,84 \%$ et la densité d'espèce de 108 individus/ha. Ceci confirme qu'on est bien en présence d'un écosystème dans un état « fortement dégradé » (Tableau 4). Sandjong Sani et al. (2013) ont obtenu les valeurs suivantes au Parc de Mozogo-Gokoro situé dans la même zone agro-écologique, mais un peu plus au Sud: 3,75 bits pour l'indice de Shannon ; 0,63 pour l'équitabilité de Piélou et 2972 individus/ha pour la densité absolue. Ces valeurs montrent que ce dernier parc est dans un état «modérément dégradé ». Les différences relevées ici montrent que malgré son potentiel, le Parc National de Waza est sous haute pression du fait: (i) de sa position géographique (qui le rend plus vulnérable aux facteurs climatiques), (ii) de la superposition de plusieurs groupes d'acteurs (éleveurs, les chasseurs, les pêcheurs, les agriculteurs et les artisans) ayant des objectifs différents (Kabelong Banoho, 2013) et (iii) de l'afflux massif des populations venant des zones plus affectées par les attaques des groupes terroristes plus au nord (celles-ci sont se sont installées à quelques kilomètres du Par cet n'hésitent pas à entrer dans cette aire protégée pour prélever illégalement les ressources ligneuses).

Orou Wari et al. (2020) ont obtenu les valeurs suivantes dans l'étude de végétation ligneuse de Malanville au nord du Bénin : 0,83 à 1,64 bits pour l'indice de Shannon; de 0,48 à 0,63 pour l'équitabilité de Pielou et de 6,56 à 59,25 individus /ha pour la densité. Ces valeurs témoignent d'un écosystème beaucoup moins diversifié que le PNW. Les faibles valeurs de diversité de la végétation ligneuse urbaine de Malanville s'expliquent par les conditions climatiques et édaphiques peu favorables à la survie de plusieurs espèces végétales. A cela s'ajoutent la divagation des animaux et l'absence des initiatives locales de plantation et de conservation des arbres.

\section{Structure verticale du peuplement}

La structure verticale de notre site d'étude montre une dominance des espèces de moyenne et de grande hauteur. Ce résultat est contraire à celui trouvé par Tchobsala et al. (2010), Kabelong Banoho (2013), Sandjong Sani et al. (2013) et Jiagho et al. (2016) qui ont trouvés des structures en $\mathrm{L}$ dans des écosystèmes similaires. Pour Tchobsala et al. (2010) et Jiagho et al. (2016), la structure en « $\mathrm{L}$ » a été observée en zone soudanienne à Ngaoundéré au Cameroun et à la périphérie du PNW et ceci est considérée comme un indice de dégradation. Dans le cadre de cette étude, la prépondérance des individus de taille moyenne ou haute est une caractéristique de la maturité de cet écosystème appartenant à une zone écologique où la trajectoire des peuplements aboutit généralement à la domination d'arbustes (steppes ou savanes arbustives) correspondant aux espèces sahélo soudaniennes ou soudano-sahélienne (Sandjong Sani et al., 2013).

\section{Impact des perturbations anthropiques}

Les indices d'activités anthropiques à savoir la coupe des branches (émondage), la coupe de bois, le pâturage et la mortalité dans le parc ont été relevés au cours de cette étude. La présence de ces indices témoigne des perturbations écologiques constatées. Le taux de mortalité est de 2,96\%. Ce taux de mortalité comparé à celui de (Adjonou et al., 2009) qui est de 21,2\% dans les forêts claires à Anogeissus leiocarpus du Parc National OtiKe'ran au Nord-Togo. Cette valeur très élevée au Nord-du Togo est due à une plus grande influence des changements climatiques couplée à la présence humaine et qui se traduisent par une mortalité anormalement accrue des arbres (taux de mortalité voisin), surtout au niveau des individus de petits diamètres. Le taux de mortalité obtenue lors de 
notre étude $(2,96 \%)$ est à priori faible et sans conséquence pour l'écosystème, mais la combinaison d'autres facteurs tels que la coupe du bois, le coiffage des arbres à la recherche du pâturage en saison sèche, la construction des cases traditionnelles à l'aide des jalons et la terre battue et la coupe du bois de chauffe constituent à moyen ou long terme un risque de dégradation plus accentué de cet écosystème.

\section{Conclusion}

Afin d'aider à un meilleur aménagement du PNW relativement peu connu sur le plan botanique, il était incontournable de disposer des données fiables et actualisées sur la flore et la végétation de ce parc. C'est sur cet objectif que se focalisait cette étude. Les résultats montrent un certain potentiel de la flore ligneuse qui reste modérément diversifiée, malgré les pressions auxquelles elle fait face. Le nombre d'espèces, la densité absolue, les différents indices de diversité, la structure verticale et horizontale de la population confirment cette situation. Malheureusement, ce potentiel est menacé de dégradation au regard des influences diverses, principalement d'origine anthropique et naturelle. Il est important de prendre des mesures idoines de protection et de restauration de cet écosystème pour ralentir le front actuel de dégradation qui menace l'intégrité du Parc.

\section{CONFLIT D'INTERETS}

Les auteurs déclarent qu'il n'y'a aucun conflit d'intérêts dans le cadre de cette étude.

\section{CONTRIBUTIONS DES AUTEURS}

ERJ a été a été en charge de la conception de la méthodologie, de la supervision du travail d'inventaire, ainsi que dans l'analyse et le traitement des données. Il a ensuite conçu, sur le base du rapport de l'étude cet article conformément aux orientations de la revue. LPRKB a participé à la conception de la méthodologie, conduit les inventaires sur le terrain, assuré le dépouillement, la compilation des données d'inventaires, participé à l'analyse et à la rédaction du rapport et de l'article. RAF a participé à la conception de la méthodologie, ainsi qu'à l'analyse et interprétation des données; a également participé à la relecture de l'article en s'assurant qu'il cadre avec les exigences de la revue.

\section{REMERCIEMENTS}

L'étude rentre dans le cadre de l'approfondissement des travaux de recherche des 2 auteurs dans cette zone qui vise à mieux renforcer les connaissances biologiques de la zone soudano-sahélienne du Cameroun. Les remerciements les plus au Conservateur du Parc National de Waza (André Ndjidda) et son équipe, ainsi qu'à l'équipe de l'UICN travaillant dans la région pour l'appui logistique précieux qu'ils ont apporté dans le cadre de cette étude.

\section{REFERENCES}

Adjonou K, Bellefontaine R, Kokou K. 2009. Les forêts claires à Anogeissus leiocarpus du Parc national Oti-Ke'ran au NordTogo : structure, dynamique et impacts des modifications climatiques. Sécheresse, 20(4): 394-6.

Bellefontaine R. 1997. Synthèse des espèces des domaines sahélien et soudanien qui se multiplient naturellement par voie végétative, pp. 95-104. Actes de l'Atelier «Fonctionnement et gestion des écosystèmes forestiers contractés sahéliens», ORSTOM - CIRAD Niamey.

Cisse A, Ouattara M, N'Guessan EA, Abrou JEN. 2020. Diversité végétale et usages des plantes dans une zone de savane soudanienne: cas de la localité de Ferkessédougou (Nord, Côte d'Ivoire). Int. J. Biol. Chem. Sci., 14(8): 2807-2825. DOI:

https://dx.doi.org/10.4314/ijbcs.v14i8.13

Djoufack-Manetsa V. 2011. Étude multi échelles des précipitations et du couvert 
végétal au Cameroun : Analyses spatiales, tendances temporelles, facteurs climatiques et anthropiques de variabilité du NDVI. Thèse de Doctorat, Université de Yaoundé I-Université de Bourgogne, $303 \mathrm{p}$.

FFEM. 2007. Méthodologie d'inventaire de produits forestiers ligneux valorisables pour une gestion durable. FFEM/ WWF/ CIRAD, Madagascar, $22 \mathrm{p}$.

Gnoumou A, Salfo S, Thiombiano A. 2020. Les groupements végétaux de la réserve de la Comoé-Léraba : caractérisation et impact des sols sur leur distribution. Int. J. Biol. Chem. Sci., 14(9): 3168-3187. DOI:

https://dx.doi.org/10.4314/ijbcs.v14i9.16

Henry M, Salis A, Asante AW. 2009. Rapport final : formation des cadres du Ministère de l'Environnement et du Cadre de Vie en comptabilité carbone. UICN, Kombissiri, Burkina Faso, 35 p.

Honvou SHS, Aboh BA, Sewade C, Teka O, Gandonou BC, Oumorou M, Sinsin B. 2021. Diversité floristique, structure et distribution des groupements végétaux des parcours d'accueil des transhumants dans la Basse et Moyenne Vallée de l'Ouémé au Bénin. Int. J. Biol. Chem. Sci., 15(1): 81-96. DOI: https://dx.doi.org/10.4314/ijbcs.v15i1.8

Jiagho ER. 2009. Les systèmes d'exploitation des ressources naturelles à la périphérie du Parc National de Waza (Cameroun): Enjeux, contraintes et opportunités pour le développement durable. Mémoire de Master II, Université Maine Le MansLaval, Laval , 72 p.

Jiagho ER, Zapfack L, Kabelong Banoho LPR, Tsayem-Demaze M, Corbonnois J, Tchawa P. 2016. Diversité de la flore ligneuse à la périphérie du Parc national de Waza (Cameroun)». VertigO - Revue Electronique en Sciences de l'Environnement, 16(1). DOI: 10.4000/vertigo. 17249
Jiagho ER. 2018. Flore et végétation ligneuse à la périphérie du Parc National de Waza: Dynamiques et implications pour une meilleure gestion. Thèse de Doctorat, Université du Mans (France) et du Doctorat/PhD de l'Université de Yaoundé I (Cameroun), Yaoundé - Mans, 355 p.

Kabelong Banoho LPR. 2013. Influences des activités anthropiques sur la ressource ligneuse dans la périphérie du Parc National de Waza. Mémoire de Master Professionnel, Université de Yaoundé I, Cameroun, $92 \mathrm{p}$.

Kent M, Coker P. 2003. Vegetation Description and Analysis: A Pratical Approch (ed). Belhaven Press: London.

Marcon E. 2011. Mesures de la Biodiversité, Ecologie des Forêts de Guyane. CNRS/CIRAD/INRA, Paris, $42 \mathrm{p}$.

Mbaiyetom H, Avana Tientcheu ML, Tchamba Ngankam M, Wouokoue Taffo JB. 2021. Diversité floristique et structure de la végétation ligneuse des parcs arborés de la zone soudanienne du Tchad. Int. J. Biol. Chem. Sci., 15(1): 68-80. DOI: https://dx.doi.org/10.4314/ijbcs.v15i1

MINFOF. 2014. Plan d'aménagement du Parc National de Waza 2015 - 2019. MINFOF, Yaoundé-Cameroun, 107 p.

Mvondo JP, Demba H, Oyo P, Longh HD. 2003. Inondation et fonctionnement d'un écosystème: bésoin de recherche pour la plaine du logone, resultat d'un séminaire organisé du 23 au 25 janvier 2002 à Maroua. CEDC/CML/IUCN, MarouaCameroun, $210 \mathrm{p}$.

Orou Wari B, Zakari S, Djaouga M, Toko Imorou I, Yabi I, Tente BAH, Djego JG. Diversité et structure de la végétation ligneuse dans la ville de Malanville au Nord-Bénin. Int. J. Biol. Chem. Sci., 15(1): $\quad$ 129-143. DOI: https://dx.doi.org/10.4314/ijbcs.v15i1.12

Picard N. 2006. Projet de développement rural participatif dans le moyen Atlas Central (projet Khénifra). CIRAD, France, 43 p. 
Sandjong Sani R, Ntoupka M, Adamou I, Vroumsia T. 2013. Etude écologique du Parc National de Mozogo-Gokoro (Cameroun): prospections préliminaires de la flore ligneuse et du sol pour sa conservation et son aménagement. Int. J. Biol. Chem. Sci., 7(6): 2434-2449. DOI: http://dx.doi.org/10.4314/ijbcs.v7i6.21

Tchobsala, Amougou A, Mbolo M. 2010. Impact of wood cut on the structure and floristic diversity of vegetation in the peri-urban zone of Ngaoundéré (Cameroon). Journal of Ecology and the Natural Environment, 2(11): 235-258.

UICN/CBLT. 2007b. Plan de gestion de la plaine d'inondation de Waza Logone. Document produit dans le cadre des Projets Pilotes UICN-CBLT. UICN, Maroua-Cameroun, $172 \mathrm{p}$.
Wafo G. 2008. Les aires protégées de l'Extrême-Nord Cameroun entre politiques de conservation et pratiques locales. Thèse de Doctorat en Géographie-Aménagement-

Environnement, Université d'Orléans, Orléans, $325 \mathrm{p}$.

White L, Edwards A. 2000. Conservation en Forêt Pluviale Africaine: Méthodes de Recherches Pratiques. Wildlife Conservation Society : New York.

Zapfack L. 2005. Impact de l'agriculture itinérante sur brulis sur la biodiversité végétale et la séquestration du carbone. Thèse de Doctorat d'Etat-ès-Sciences, Université de Yaoundé I, Cameroun, 249 p. 